\title{
Mechanical properties of monolayer GaS and GaSe crystals
}

\author{
M. Yagmurcukardes, ${ }^{1, *}$ R. T. Senger, ${ }^{1}$ F. M. Peeters,${ }^{2}$ and H. Sahin ${ }^{3, \dagger}$ \\ ${ }^{1}$ Department of Physics, Izmir Institute of Technology, 35430 Izmir, Turkey \\ ${ }^{2}$ Department of Physics, University of Antwerp, Groenenborgerlaan 171, B-2020 Antwerp, Belgium \\ ${ }^{3}$ Department of Photonics, Izmir Institute of Technology, 35430 Izmir, Turkey
}

(Received 21 June 2016; revised manuscript received 4 November 2016; published 5 December 2016)

\begin{abstract}
The mechanical properties of monolayer GaS and GaSe crystals are investigated in terms of their elastic constants: in-plane stiffness $(\mathrm{C})$, Poisson ratio $(v)$, and ultimate strength $\left(\sigma_{U}\right)$ by means of first-principles calculations. The calculated elastic constants are compared with those of graphene and monolayer $\mathrm{MoS}_{2}$. Our results indicate that monolayer $\mathrm{GaS}$ is a stiffer material than monolayer GaSe crystals due to the more ionic character of the Ga-S bonds than the Ga-Se bonds. Although their Poisson ratio values are very close to each other, 0.26 and 0.25 for $\mathrm{GaS}$ and $\mathrm{GaSe}$, respectively, monolayer $\mathrm{GaS}$ is a stronger material than monolayer GaSe due to its slightly higher $\sigma_{U}$ value. However, GaS and GaSe crystals are found to be more ductile and flexible materials than graphene and $\mathrm{MoS}_{2}$. We have also analyzed the band-gap response of $\mathrm{GaS}$ and GaSe monolayers to biaxial tensile strain and predicted a semiconductor-metal crossover after $17 \%$ and $14 \%$ applied strain, respectively, for monolayer GaS and GaSe. In addition, we investigated how the mechanical properties are affected by charging. We found that the flexibility of single layer GaS and GaSe displays a sharp increase under $0.1 e /$ cell charging due to the repulsive interactions between extra charges located on chalcogen atoms. These charging-controllable mechanical properties of single layers of $\mathrm{GaS}$ and GaSe can be of potential use for electromechanical applications.
\end{abstract}

DOI: 10.1103/PhysRevB.94.245407

\section{INTRODUCTION}

Layered bulk materials that consist of two-dimensional (2D) very thin sheets, stacked with weak out-of-plane van der Waals (vdW) interaction, have been the focus of interest for more than a century $[1,2]$. As presented by review studies [3-7], when a layered material is thinned from bulk to monolayer, it can exhibit enhanced and different physical properties. Post-transition-metal chalcogenides (PTMCs) are important examples of such vdW layered materials having the chemical formula $M X$ where $M$ stands for post-transition metal and $X$ for chalcogenide atom [8-11]. GaS and GaSe are two emerging members of PTMCs. As in most of the monolayer materials, monolayer $\mathrm{GaS}$ and $\mathrm{GaSe}$ possess different electronic properties than their bulk form. In particular, thinning the GaSe to its monolayer form results in a direct-toindirect band-gap transition.

$\mathrm{GaS}$ and $\mathrm{GaSe}$ structures are layered semiconductors in their bulk form possessing direct or indirect gap character depending on the type of chalcogenide atom. They consist of weakly bound atomic thin layers in which there are two sheets of Ga layers sandwiched between chalcogenide layers (in order of $X-\mathrm{Ga}-\mathrm{Ga}-X$, where $X=\mathrm{S}$ or Se). The intralayer bonding of PTMCs has strong covalent character, while the interlayer interaction has weak vdW character. Bulk GaSe crystal was reported to be a direct-gap semiconductor with a band gap of $2.0 \mathrm{eV}$, while bulk GaS was found to be an indirect-gap semiconductor with a band gap of $2.4 \mathrm{eV}[12,13]$. In recent years large area ultrathin layers of $\mathrm{GaS}$ and $\mathrm{GaSe}$ crystals were successfully synthesized on $\mathrm{SiO}_{2} / \mathrm{Si}$ substrates by using micromechanical cleavage technique [14-18]. Chen et al.

\footnotetext{
*mehmetyagmurcukardes@iyte.edu.tr

†hasansahin@iyte.edu.tr
}

studied theoretically the electronic and magnetic properties of substitutionally doped monolayer $\mathrm{GaS}$ and found that the $\mathrm{N}$ atom is the most promising candidate for $p$-type doping among nonmetal and transition-metal dopants. They also investigated the electronic and magnetic properties of native defects in $\mathrm{GaS}$ monolayer and found a half-metallic behavior for $\mathrm{Ga}$ vacancy $[19,20]$. Li et al. studied the interlayer orientations and stackings of 2D bilayer GaSe crystals and reported that the rotational energy barriers increase as the size of the system increases [21]. Moreover, Zhou et al. studied the second-harmonic generation in layered GaSe crystals and found that the strongest second-harmonic generation intensity is observed for GaSe among all 2D crystals [22].

Although the electronic, magnetic, and optical properties of monolayer $\mathrm{GaS}$ and $\mathrm{GaSe}$ crystals have been studied extensively, their mechanical properties are still unknown. Therefore, we perform a comparative study of the mechanical properties of single layer crystal structures of $\mathrm{GaS}, \mathrm{GaSe}$, graphene, and $\mathrm{MoS}_{2}$ in terms of their elastic constants. In addition, the effects of charging the system on the mechanical properties of monolayer $\mathrm{GaS}$ and $\mathrm{GaSe}$ are investigated.

The paper is organized as follows: Details of the computational methodology are given in Sec. II. Structural and electronic properties of monolayers of graphene, $\mathrm{MoS}_{2}, \mathrm{GaS}$, and GaSe are presented in Sec. III. In Sec. IV, the mechanical properties of these monolayers and the effect of charging on their elastic constants are discussed in detail. Finally, we conclude in Sec. V.

\section{COMPUTATIONAL METHODOLOGY}

For our first-principles calculations, we employed the plane-wave basis projector augmented wave method in the framework of density-functional theory (DFT). For the exchange-correlation potential, the generalized gradient 

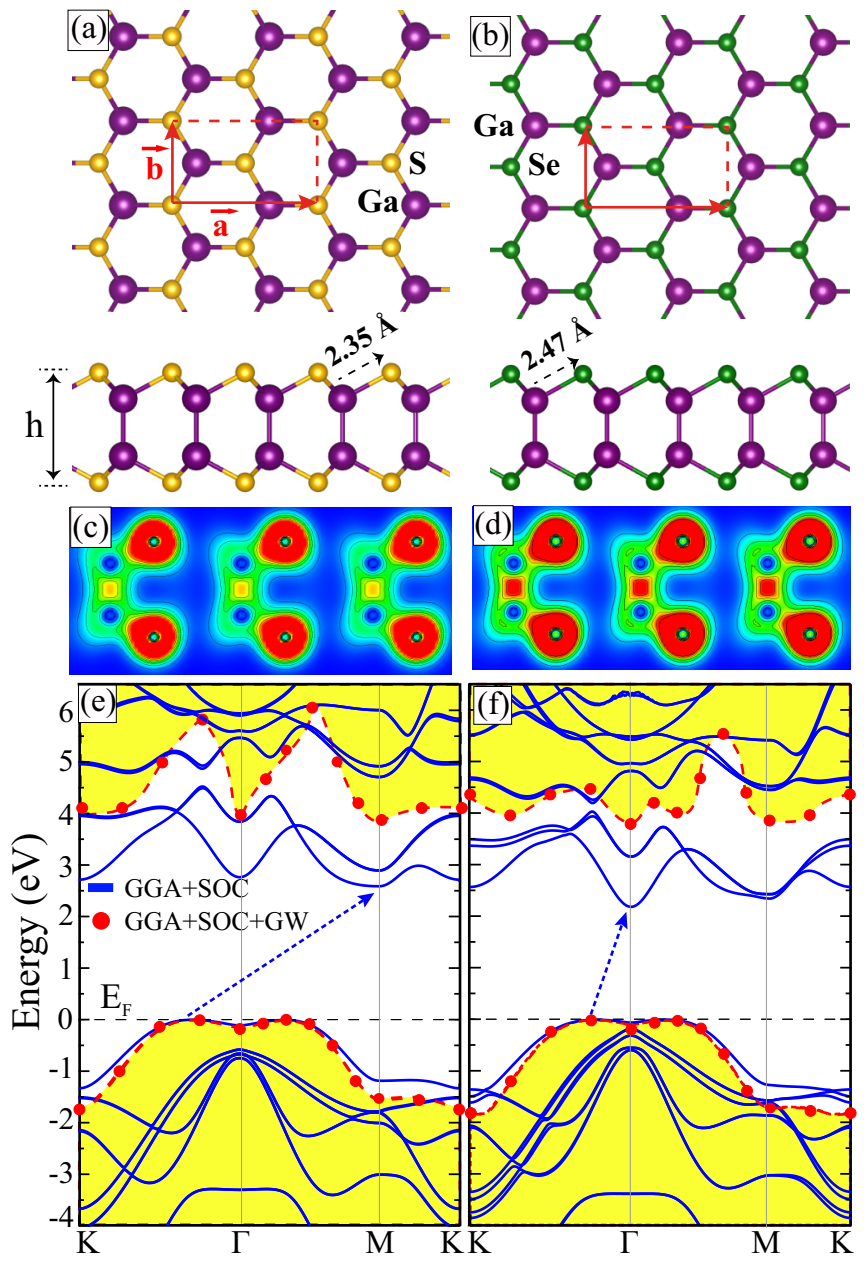

FIG. 1. Top and side view of monolayer (a) GaS and (b) GaSe. Red dashed lines represent the rectangular unit cell and $a$ and $b$ are the lattice vectors. $h$ is the thickness of the monolayer GaS and GaSe crystals. The charge distribution on the individual atoms for monolayer (c) GaS and (d) GaSe are shown in side view. Increasing charge density is shown by a color scheme from blue to red with linear scaling between zero (blue) and $6.7 e / \AA^{3}$ (red). (e) and (f) show the calculated energy-band structure within GGA+SOC and $\mathrm{GGA}+\mathrm{SOC}+\mathrm{GW}$ approximations for monolayer $\mathrm{GaS}$ and $\mathrm{GaSe}$, respectively. The Fermi energy $\left(E_{F}\right)$ level is set to the valence band maximum. The red dashed lines indicate the $\mathrm{GW}$ band structure, while blue lines indicate the indirect GGA+SOC band structure.

approximation (GGA) in the Perdew-Burke-Ernzerhof form [24,25] was employed with the inclusion of spin-orbit coupling (SOC) as implemented in the Vienna $a b$ initio simulation package (VASP) [26,27]. The vdW correction to the GGA functional was included by using the DFT-D2 method of Grimme [28]. Analysis of the charge transfers in the structures was determined by the Bader technique [29].

Electronic and geometric relaxations of the structures were performed by considering a parallelogram unit cell containing two atoms (for graphene), three atoms (for $\mathrm{MoS}_{2}$ ), or four atoms (for $\mathrm{GaS}$ and GaSe structures) (see Fig. 1). In addition, for mechanical calculations a rectangular unit cell was considered for all monolayers. The energy cut-off value for the plane-wave basis set was taken to be $500 \mathrm{eV}$. The total energy was minimized until the energy variation in successive steps became less than $10^{-5} \mathrm{eV}$ in the structural relaxation and the convergence criterion for the Hellmann-Feynman forces was taken to be $10^{-4} \mathrm{eV} / \AA$. The minimum energy was obtained by varying the lattice constant and by reducing the pressure below 1 kbar in all directions. $27 \times 27 \times 1 \Gamma$-centered k-point sampling is used for the primitive unit cell. The broadening of the density of states was taken to be $0.05 \mathrm{eV}$. All calculations, including the charged systems, were performed with spin polarized case.

The optical band gaps of monolayer GaS and GaSe were calculated by solving the Bethe-Salpeter equation on top of the $\mathrm{GW}\left(\mathrm{G}_{0} \mathrm{~W}_{0}\right)$ calculation including the SOC. For these calculations $12 \times 12 \times 1 \Gamma$-centered $\mathbf{k}$-point sampling was used and 160 bands were included in our calculations. The cutoff energy for the plane waves was chosen to be $400 \mathrm{eV}$.

\section{STRUCTURAL AND ELECTRONIC PROPERTIES}

Monolayers of $\mathrm{GaS}$ and GaSe crystals consist of four-atom layers in the order $X-\mathrm{Ga}-\mathrm{Ga}-X$ [see Figs. 1(a) and 1(b)] where $X$ stands for $\mathrm{S}$ or $\mathrm{Se}$ atoms. The calculated lattice parameters are 3.58 and $3.75 \AA$ for monolayers of $\mathrm{GaS}$ and GaSe structures, respectively. These results are compatible with the calculated $\mathrm{Ga}-X$ bond lengths, i.e., 2.35 and $2.47 \AA$ for the Ga-S and Ga-Se bonds, respectively (2.41 $\AA$ for the Mo-S bond in monolayer $\mathrm{MoS}_{2}$ ). The thicknesses of the monolayers were calculated to be 4.66 and $4.82 \AA$ for $\mathrm{GaS}$ and GaSe, respectively. In both monolayers charge donation occurs from $\mathrm{Ga}$ to chalcogenide atoms. In monolayer $\mathrm{GaS}$ each $\mathrm{Ga}$ atom donates $0.8 e$ to each $\mathrm{S}$ atom which indicates the strongly ionic character of the Ga-S bond [see Fig. 1(c)]. As seen in Fig. 1(d), the amount of charge depletion from $\mathrm{Ga}$ to Se atoms decreases to $0.6 e$ per atom in monolayer GaSe which indicates the mostly covalent character of the Ga-Se bond. This is because of the charge depletion between Ga atoms which is seen by the small red area in Fig. 1(d). The work function values were calculated as 6.10 and $5.59 \mathrm{eV}$ for $\mathrm{GaS}$ and $\mathrm{GaSe}$, respectively. The work function of monolayer $\mathrm{MoS}_{2}$ is smaller than that of monolayer $\mathrm{GaS}$ with the value of $5.88 \mathrm{eV}$. Monolayer GaSe has a work function of $5.59 \mathrm{eV}$, which is lower than that of $\mathrm{GaS}$ due to the higher ionizations energy of the $\mathrm{S}$ atom as compared to the Se atom. For both of the monolayers, work functions decrease upon charging the structures. When $0.1 e$ per primitive cell is added, extra charges accumulate to $p_{z}$ orbitals of chalcogenide atoms. Thus, work function shows a rapid decrease since it is calculated from the surface of the material. However, when $0.1 e$ per primitive cell is added, now the extra charges are depleted to inside the monolayers. Therefore, the work function shows a decrease with small amount of energy difference. In addition, charging the structures expands the lattice and thus, the lattice constant tends to increase upon charging. The cohesive energy decreases for larger structures. Therefore, the cohesive energies show decreasing trend upon charging the system.

Although they exhibit different semiconducting characters in their bulk forms, monolayers of $\mathrm{GaS}$ and $\mathrm{GaSe}$ are indirect band-gap semiconductors. Their valence band maximum (VBM) lies between the $K$ and $\Gamma$ points in the Brillouin zone (BZ). However, it is important to note that the valence 


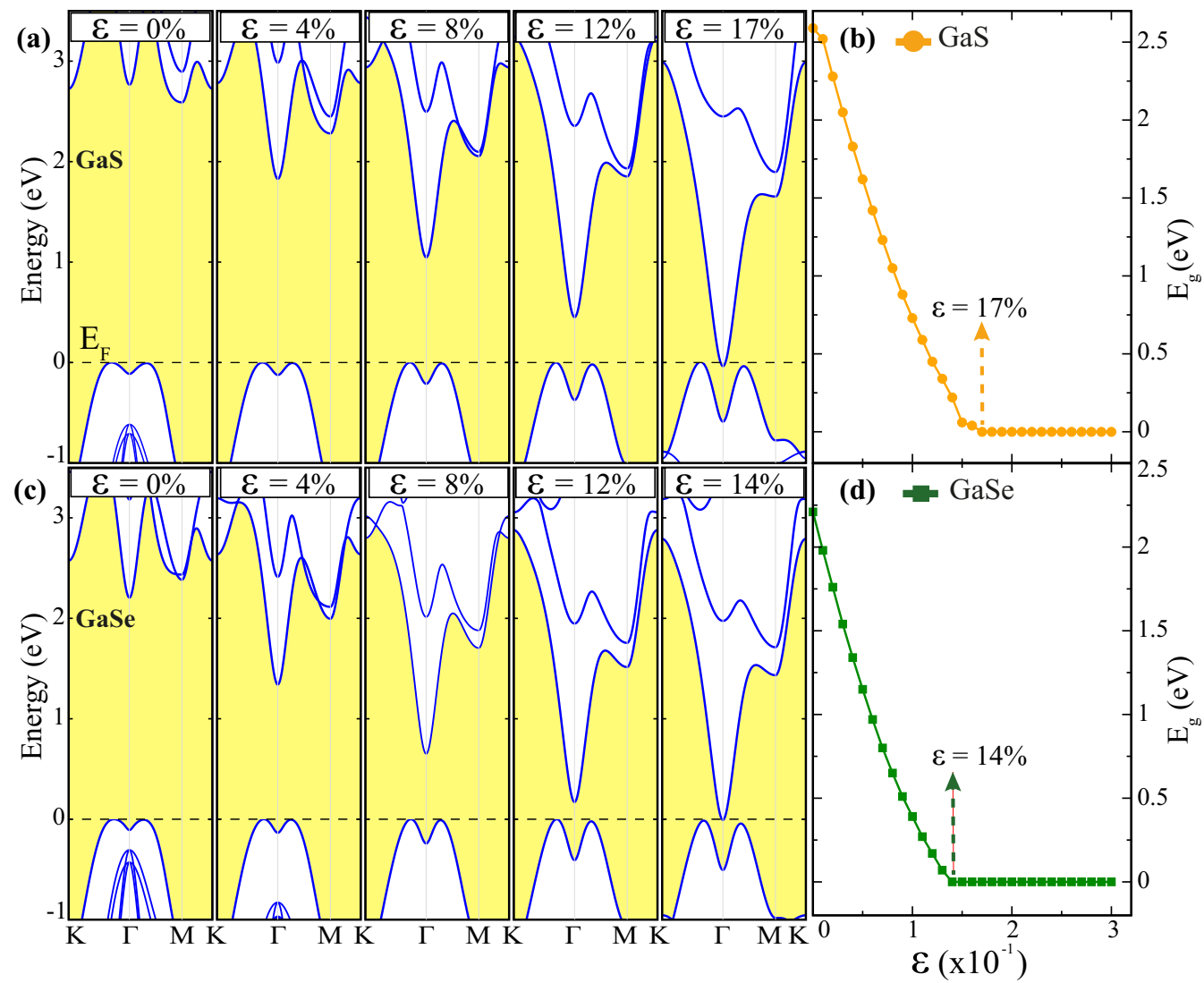

FIG. 2. Calculated energy-band structures within GGA for different applied strain values for monolayer (a) GaS and (c) GaSe. The Fermi energy $\left(E_{F}\right)$ level is set to the VBM of each structure. The change in the band gap with applied strain for monolayer (b) GaS and (d) GaSe.

band edge in between $\Gamma$ and $M$ points differs only by $6 \mathrm{meV}$ energy than the VBM of the monolayers. The conduction band minimum (CBM) resides at the $M$ point in monolayer $\mathrm{GaS}$, while it resides at the $\Gamma$ point in monolayer GaSe [see Figs. 1(e) and 1(f)]. The calculated GGA+SOC band gaps are 2.59 and $2.18 \mathrm{eV}$ for monolayer $\mathrm{GaS}$ and GaSe, respectively. These values of the band gap will increase when we perform GW calculation within SOC. Our calculated GW band gaps are 3.88 and $3.68 \mathrm{eV}$ for monolayer $\mathrm{GaS}$ and $\mathrm{GaSe}$, respectively.

Before the detailed investigation of the mechanical characteristics of monolayer $\mathrm{GaS}$ and $\mathrm{GaSe}$, we show how the electronic structure responds against applied biaxial strain. In order to investigate the response of the electronic band structure of the monolayers to applied biaxial strain, we considered the BZ of the rectangular unit cell shown in Fig. 2. As seen in Figs. 2(b) and 2(d), the band gap of the two monolayers decreases monotonously with increasing applied strain. The electronic band structure of GaS displays metallic behavior firstly at $\varepsilon=17 \%$. The indirect-gap character of $\mathrm{GaS}$ is not affected by the applied strain, only the value of the gap decreases. As the applied strain increases, the energy difference between two valence band edges also increases. A similar shift in VBM of hexagonal aluminium nitride (h-AIN) was reported by Bacaksiz et al. [30]. The VBM of GaS consists of $p_{z}$ orbitals of the $\mathrm{Ga}$ and $\mathrm{S}$ atoms. Thus, the out-of-plane orbitals are not affected by the applied in-plane strain. Differing from the atomic orbital character of VBM,
CBM consists of both a $p_{z}$ orbital of the $\mathrm{S}$ atom and $p_{x}, p_{y}$ orbitals of the $\mathrm{Ga}$ atom. The in-plane orbitals of the $\mathrm{Ga}$ atom are strongly affected by the in-plane strain and thus, the energy of CBM decreases [see Fig. 2(a)]. The same behavior of band structure can be seen in monolayer GaSe under applied biaxial strain. The metallic behavior of GaSe is first seen at $\varepsilon=14 \%$, which is smaller than that of GaS. Although we reported the critical strain values for semiconductor-metal transition, it should be noted that these values depend also on the value of the band gap. If one considers the GW gaps or band gaps calculated within HSE06, critical strain values may be found to be higher. Since the VBM of GaSe consists of $p_{z}$ orbitals of $\mathrm{Ga}$ and Se atoms, it is also unaffected by the applied strain. Only a small energy difference occurs between the valence band edges at $K-\Gamma$ and $\Gamma-M(6 \mathrm{meV})$. However, the CBM of GaSe consists of an $s$ orbital of Ga and $p_{z}$ and $s$ orbitals of $\mathrm{Se}$ atoms.

\section{MECHANICAL PROPERTIES}

The elastic properties of homogeneous and isotropic materials can be represented by two independent constants: the in-plane stiffness $C$ and the Poisson ratio $v$. To examine the brittleness or ductility of materials, the fracture strain values must be obtained from the stress-strain curve. In the following we mind a comparative investigation of the mechanical properties of monolayer $\mathrm{GaS}, \mathrm{GaSe}$, graphene, and $\mathrm{MoS}_{2}$ crystals in terms of these elastic constants. 
For the determination of the elastic constants of the four different monolayer structures, the rectangular unit cell shown in Fig. 1(a) was first optimized and then a $2 \times 2$ supercell (containing 32 atoms) was considered. The strains $\varepsilon_{x}$ and $\varepsilon_{y}$ were applied to the monolayer crystals by varying the lattice constants along the $x$ and $y$ directions. The strain parameters $\varepsilon_{x}$ and $\varepsilon_{y}$ were varied between \pm 0.02 with a step size of 0.01 . For this purpose three different sets of data were calculated: (i) $\varepsilon_{y}=0$ and $\varepsilon_{x}$ varying, (ii) $\varepsilon_{x}=0$ and $\varepsilon_{y}$ varying, and (iii) $\varepsilon_{x}=\varepsilon_{y}$ varying. At each configuration, the atomic positions were fully relaxed and the strain energy, $E_{S}$, was calculated by subtracting the total energy of the strained system from the equilibrium total energy. The calculated data was fitted to the equation $E_{S}=c_{1} \varepsilon_{x}^{2}+c_{2} \varepsilon_{y}^{2}+c_{3} \varepsilon_{x} \varepsilon_{y}$, and the coefficients $\mathrm{c}_{i}$ were determined.

\section{A. In-plane stiffness}

The in-plane stiffness, $C$, is a measure of the rigidity or the flexibility of a material and depends on the geometry of the considered structure. Graphene is known to be the stiffest material among the 2D monolayers. The value of $C$ can be calculated by the formula $C=\left(1 / A_{0}\right)\left(2 c-c_{3}^{2} / 2 c\right)$ where we let $c_{1}=c_{2}=c$ due to the isotropy of the unit cell and $A_{0}$ is the strain-free area of the supercell. To understand the flexibility of monolayer $\mathrm{GaS}$ and $\mathrm{GaSe}$, it is meaningful to compare their in-plane stiffness with that of well-known 2D materials, such as graphene and $\mathrm{MoS}_{2}$. Our calculated in-plane stiffness values are 91 and $77 \mathrm{~N} / \mathrm{m}$ for $\mathrm{GaS}$ and GaSe, respectively, which are close to the value for $\mathrm{MoS}_{2}(122 \mathrm{~N} / \mathrm{m})$, but lower than that of graphene $(330 \mathrm{~N} / \mathrm{m})$. Our results for graphene and $\mathrm{MoS}_{2}$ are consistent with the reported experimental values [31-35]. Although monolayer $\mathrm{GaS}$ and GaSe have much lower in-plane stiffness values than graphene, they are stiffer than silicene and germanene [36]. The difference in the in-plane stiffness values between two Ga chalcogenides can be explained through the lattice constants and cohesive energies of two monolayers. The lattice constant of $\mathrm{GaS}$ is smaller than that of GaSe and parallel to the ionization energies of chalcogenide atoms, the cohesive energy per atom is higher for $\mathrm{GaS}$ (these two parameters, lattice constant and cohesive energy, are also in good agreement). However, when we compare the two monolayers with the same chalcogenide atom, S, monolayer $\mathrm{MoS}_{2}$ is stiffer than monolayer GaS. This can be understood when the total charge densities are analyzed; the electrons are mostly localized on the $\mathrm{S}$ atom in $\mathrm{GaS}$, while they are shared between Mo and $\mathrm{S}$ atoms in monolayer $\mathrm{MoS}_{2}$, which indicates the more covalent bond characters in $\mathrm{MoS}_{2}$.

As seen in Figs. 3(a) and 3(b), in-plane stiffness decreases with increasing lattice constant and the cohesive energy per atom. This is expected because a smaller lattice constant means a smaller bond length between the individual atoms, which indicates a higher in-plane stiffness. In contrast, as seen in Fig. 3(c), in-plane stiffness decreases with increasing work function. The work function of a material is proportional to the ionization energy of individual atoms in the material. So higher ionization energy means higher work function, which correlates with the higher in-plane stiffness.

In order to tune the elastic properties of monolayer $\mathrm{GaS}$ and GaSe crystals, extra electrons were added to the monolayers.

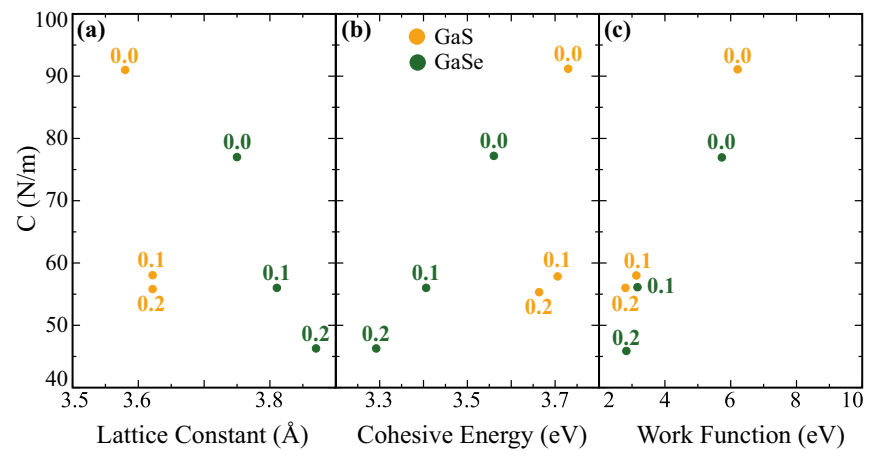

FIG. 3. In-plane stiffness values: (a) lattice constant, (b) cohesive energy per atom, and (c) work function of monolayer $\mathrm{GaS}$ and $\mathrm{GaSe}$ crystals. The number near the symbols gives the amount of added charge per unit cell.

We considered two different amounts of charges, 0.1 and $0.2 e /$ cell, respectively. For monolayer $\mathrm{GaS}$, we find that the in-plane stiffness decreases to 58 and $56 \mathrm{~N} / \mathrm{m}$ for 0.1 and $0.2 e /$ cell charging. In the case of monolayer $\mathrm{GaSe}$, the trend in the in-plane stiffness is the same. The 0.1 and $0.2 e /$ cell-charged cases decrease the value of $C$ to 54 and $46 \mathrm{~N} / \mathrm{m}$, respectively.

As shown in Fig. 4 when $0.1 e /$ cell is added, the extra charges are depleted to the $p_{z}$ orbitals of $\mathrm{S}$ and Se atoms in both monolayers. Therefore, following the increase in $\mathrm{Ga}-X$ bond lengths, the in-plane stiffness shows a sharp decrease with $0.1 e$ charging. However, $\mathrm{GaS}$ and $\mathrm{GaSe}$ have a different behavior for further charging. Our charge density difference analysis shown in Fig. 4 reveals that for $0.2 e$ /cell charged GaS, the additional charges are donated to both in-plane and out-of-plane orbitals (see Fig. 4). However, in the case of monolayer GaSe additional charges are accumulated mostly to out-of-plane orbitals of the $\mathrm{Se}$ atoms. Therefore, in-plane-located extra charges of $\mathrm{GaS}$ makes it stiffer than GaSe even at $0.2 e$ charging and decrease in in-plane stiffness of $\mathrm{GaS}$ is less than $\mathrm{GaSe}$.
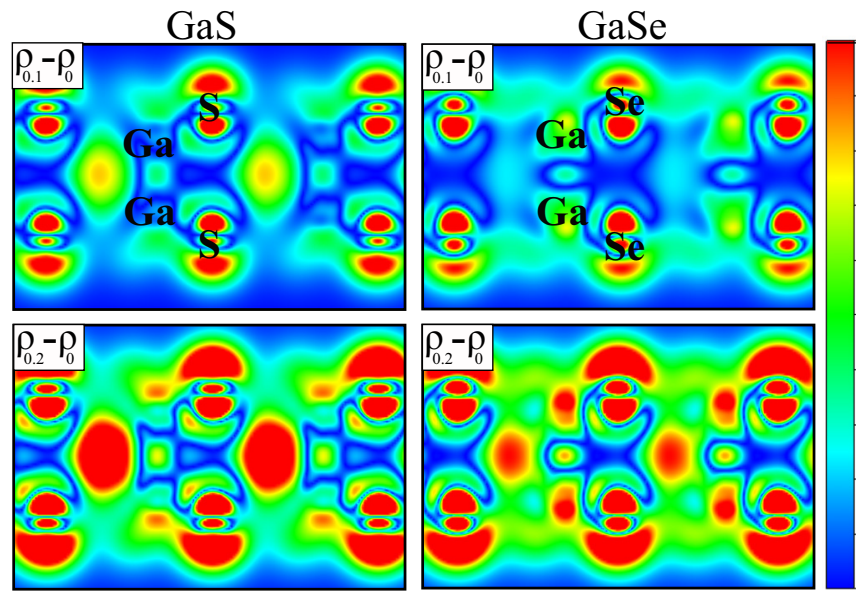

FIG. 4. Total charge density difference between the bare and 0.1 charged $\left(\rho_{0.1}-\rho_{0}\right)$, and 0.2 charged $\left(\rho_{0.2}-\rho_{0}\right)$ cases for monolayer GaS and GaSe. Increasing charge density is shown by a color scheme from blue to red with linear scaling between zero (blue) and maximum (red) charge. 


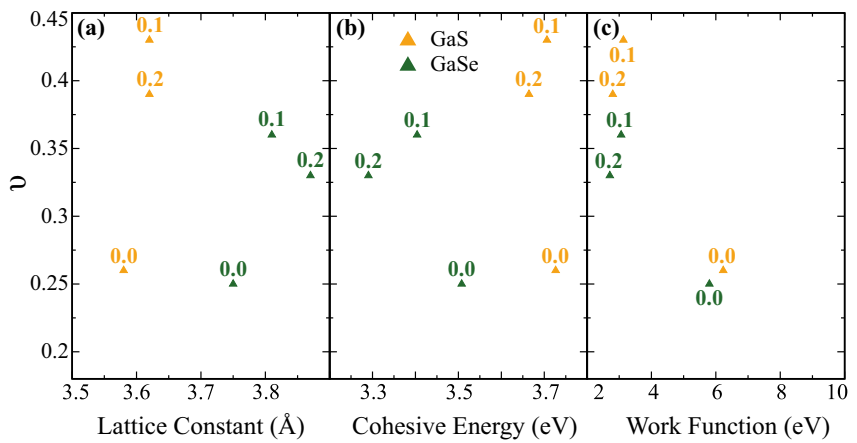

FIG. 5. Poisson ratio values: (a) lattice constant, (b) cohesive energy per atom, and (c) work function of monolayer GaS and GaSe crystals. The numbers above the symbols refer to the amount of added charge per unit cell.

\section{B. Poisson ratio}

The mechanical response of a material to an applied stress is called the Poisson ratio. It is also defined as the ratio of the transverse contraction strain to the longitudinal extension strain in the direction of the stretching force, that is, $v=$ $-\varepsilon_{\text {trans }} / \varepsilon_{\text {axial }}$. The Poisson ratio was obtained as $v=c_{3} / 2 c$. Due to the symmetry of the honeycomb lattice, the elastic constants were found to be the same along the $x$ and $y$ directions. The calculated Poisson ratio values for $\mathrm{GaS}$ and GaSe are 0.26 and 0.25 , respectively, which are nearly the same as that of monolayer $\mathrm{MoS}_{2}(0.26)$ but larger than that of graphene (0.19) (Fig. 5). This means that GaS and GaSe crystals are more sensitive to applied uniaxial strain. Indeed, for the same applied uniaxial strain, a longer contraction is found in the perpendicular direction to the applied strain direction.

In connection with the value of $C$ in the absence of charging, the Poisson ratio of the monolayer is also strongly affected. When the monolayer $\mathrm{GaS}$ is charged, it becomes more flexible as mentioned and the corresponding Poisson ratio value increases. This situation is expected because a flexible material can contract much smaller in the transverse direction when stretched along the axial direction. We found $v$ to be 0.43 and 0.39 for $\mathrm{GaS}$ and 0.36 and 0.33 for GaSe under 0.1 and $0.2 e /$ cell charging, respectively. Opposite to the trends in $C$, the Poisson ratio value first increases under charging by $0.1 e /$ cell and then decreases under charging by $0.2 e /$ cell. But in both cases the Poisson ratio is higher than those for bare monolayers. When the structures are charged, monolayers become more flexible, which indicates more extension of the lattices under small uniaxial strains. Thus, the Poisson ratio values demonstrate a rapid increase because of the lattice expansion under charging.

\section{Ultimate strength}

The ultimate strength is the maximum value of stress that a material can resist before the fracture point. This value can be directly investigated from the maximum point of the stress-strain curve of a material. For this purpose, large biaxial strain was applied to the monolayers up to the value of $\varepsilon=0.40$. As seen in Fig. 6(b), monolayer GaS and GaSe have much lower ultimate strength values than that of graphene and monolayer $\mathrm{MoS}_{2}$. Our results indicate that monolayer GaS has $\sigma_{U}=12 \mathrm{GPa}$, which is higher than that of monolayer $\mathrm{GaSe}$ which is calculated to be $10 \mathrm{GPa}$ as given in Table I. The very high value of $\sigma_{U}$ for graphene is due to the very strong $\mathrm{sp}^{2} \mathrm{C}-\mathrm{C}$ bonds. Although these two monolayers have lower $\sigma_{U}$, they can resist higher values of applied biaxial strain than graphene and $\mathrm{MoS}_{2}$. The calculated ultimate strains for GaS and GaSe are $\varepsilon_{U}=0.24$ and $\varepsilon_{U}=0.23$, respectively. They are calculated to be $\varepsilon_{U}=0.20$ and $\varepsilon_{U}=0.22$ for graphene and $\mathrm{MoS}_{2}$, respectively. These values indicate that Gas and GaSe crystals are more ductile than graphene. In addition, monolayers of $\mathrm{GaS}$ and GaSe crystals have high values of fracture strain as seen in Fig. 6(b). $\varepsilon_{F}$ values were calculated as 0.29 and 0.30 for $\mathrm{GaS}$ and $\mathrm{GaSe}$, which are larger than that of graphene (0.26) and monolayer $\mathrm{MoS}_{2}(0.24)$.
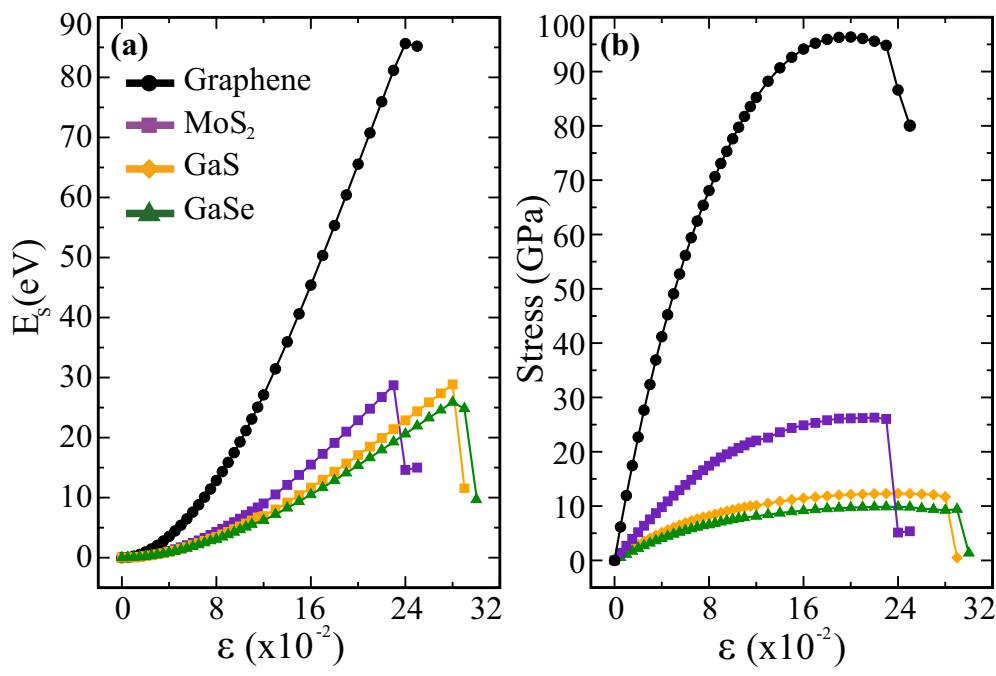
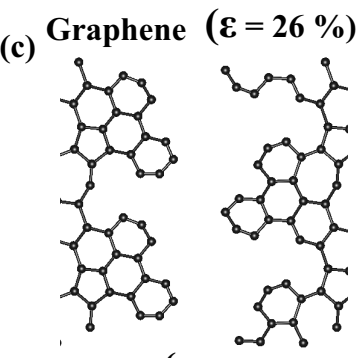

$$
\operatorname{GaS}(\varepsilon=29 \%)
$$

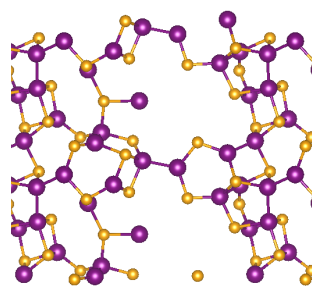

$\operatorname{MoS}_{2}(\varepsilon=24 \%)$

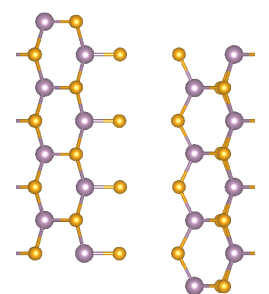

$\operatorname{GaSe}(\varepsilon=30 \%)$



FIG. 6. (a) The change of strain energy and (b) the created stress in the different monolayer materials under applied biaxial strain. (c) The geometries of each monolayer crystal at fracture strain values. 
TABLE I. The calculated ground state properties of monolayer graphene, $\mathrm{MoS}_{2}, \mathrm{GaS}$, and GaSe crystals: proper thickness $h_{p}$, magnetic state, the amount of charge received by the a chalcogenide atom $\Delta \rho$, calculated energy band gap within GGA $\left(E_{\mathrm{g}}^{\mathrm{GGA}}\right), \mathrm{SOC}\left(E_{\mathrm{g}}^{\mathrm{SOC}}\right), \mathrm{GW}\left(E_{\mathrm{g}}^{\mathrm{GW}}\right)$, and work function $\Phi$, in-plane stiffness $\mathrm{C}$, Poisson ratio $\nu$, ultimate strength $\sigma_{U}$, ultimate strain $\varepsilon_{U}$, and fracture strain $\varepsilon_{F}$.

\begin{tabular}{lccccccccccc}
\hline \hline & $\begin{array}{c}h_{p} \\
(\AA)\end{array}$ & $\begin{array}{c}\text { Magnetic } \\
\text { state }\end{array}$ & $\begin{array}{c}\Delta \rho \\
(e)\end{array}$ & $\begin{array}{c}E_{\mathrm{g}}^{\mathrm{GGA}} \\
(\mathrm{eV})\end{array}$ & $\begin{array}{c}E_{\mathrm{g}}^{\mathrm{SOC}} \\
(\mathrm{eV})\end{array}$ & $\begin{array}{c}E_{\mathrm{g}}^{\mathrm{GW}} \\
(\mathrm{eV})\end{array}$ & $\begin{array}{c}\Phi \\
(\mathrm{eV})\end{array}$ & $\begin{array}{c}\mathrm{C} \\
(\mathrm{N} / \mathrm{m})\end{array}$ & $\begin{array}{c}\sigma_{U} \\
v\end{array}$ & $\begin{array}{c}\varepsilon_{U} \\
(\mathrm{GPa})\end{array}$ & $\begin{array}{c}\varepsilon_{F} \\
(\%)\end{array}$ \\
\hline Graphene & 3.35 & $\mathrm{NM}$ & & & & & 4.40 & 330 & 0.19 & 96 & 20 \\
$\mathrm{MoS}_{2}$ & 6.15 & $\mathrm{NM}$ & 0.5 & 1.64 & 1.56 & $2.80[23]$ & 5.88 & 122 & 0.26 & 26 & 22 \\
$\mathrm{GaS}$ & 7.76 & $\mathrm{NM}$ & 0.8 & 2.59 & 2.59 & 3.88 & 6.10 & 91 & 0.26 & 12 & 24 \\
$\mathrm{GaSe}$ & 7.96 & $\mathrm{NM}$ & 0.6 & 2.21 & 2.18 & 3.68 & 5.59 & 77 & 0.25 & 10 & 23 \\
\hline \hline
\end{tabular}

\section{Dynamical instability}

The stress-strain relation can be used for extracting many mechanical parameters for a material. In order to calculate these mechanical constants and to determine the mechanical characteristics, we plot the theoretical stress-strain relation
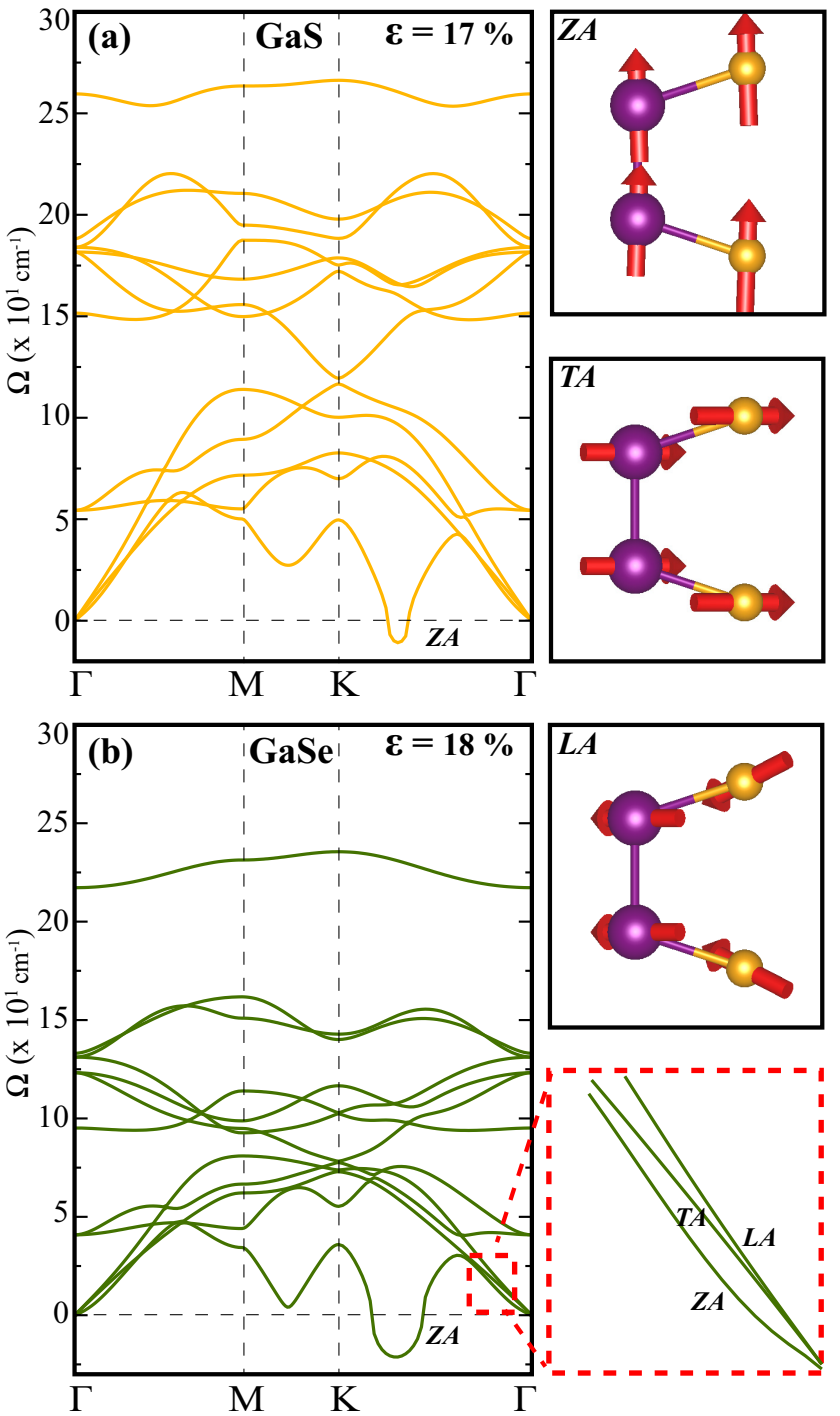

FIG. 7. Phonon-band dispersions for monolayer (a) GaS at $17 \%$ and (b) GaSe $18 \%$ biaxial strains. The three acoustic phonon modes are given in the right panel. under applied biaxial strain. To compute the stress-strain relationship, a series of incremental tensile strains are applied to the monolayers. The maximum stress point of a stress-strain curve is critical for determining the fracture strain point of the material. Beyond that point, the structure becomes elastically unstable by fracture. However, this does not mean that the material remains dynamically stable up to the fracture point at the stress- strain curve. When phonon frequencies were calculated for monolayer $\mathrm{GaS}$ and GaSe under biaxial strain, we found that $\mathrm{GaS}$ and $\mathrm{GaSe}$ crystals are no longer dynamically stable after $17 \%$ and $18 \%$ biaxial strain values, respectively.

As seen from the Fig. 7, both monolayers undergo phonon instability which is dictated by an out-of-plane soft mode. The instability occurs at the wave vector between the $K$ and the $\Gamma$ points. When related to the wavelength of this unstable acoustic phonon mode, the wave vector has a value of $0.8041 / \AA$. Thus, the higher value of the wave vector reveals the smaller value of the wavelength for which the material has instability. In addition, we found that increased biaxial strain causes the region of unstable wave vectors to grow significantly before reaching an elastically unstable strain point that corresponds to the peak of the stress-strain curve. Here the underlying mechanism can be explained through the bond angles ( $X$-Ga- $X$ bond) in the upper and the lower Ga- $X$ layers. As the applied biaxial strain is increased, this bond angle also increases and thus, the upper and lower $\mathrm{Ga}-X$ layers become more planar, which softens the out-of-plane acoustic (ZA) mode of the monolayer structures. Similar to graphene and other 2D materials, phonon instability, that stems from the out-of-plane soft mode, occurs before the elastic instability in monolayer $\mathrm{GaS}$ and $\mathrm{GaSe}$.

\section{CONCLUSION}

In conclusion, we have investigated the mechanical properties of novel 2D monolayer GaS and GaSe crystals in terms of in-plane stiffness, Poisson ratio, and ultimate strength. We compare their values with those of graphene and monolayer $\mathrm{MoS}_{2}$. Our results revealed that due to the more ionic character of the $\mathrm{Ga}-\mathrm{S}$ bond as compared to the $\mathrm{Ga}-\mathrm{Se}$ one, monolayer $\mathrm{GaS}$ is a slightly stiffer material than monolayer GaSe. Calculated stress-strain curves for each monolayer crystal indicate that they have smaller critical strength values than graphene and monolayer $\mathrm{MoS}_{2}$ but higher ultimate strains. In addition, monolayer GaS and GaSe crystals have higher fracture strain 
values when compared to graphene and monolayer $\mathrm{MoS}_{2}$, which is a signiture of their ductility. By further analysis, decreasing trends in band-gap values of monolayer $\mathrm{GaS}$ and GaSe crystals were found under biaxial strain at $17 \%$ and $14 \%$ for $\mathrm{GaS}$ and $\mathrm{GaSe}$, respectively. Our results on charged structures indicate that extra charges are depleted to the out-of-plane orbitals, which expands the structures by repulsive interaction resulting in the reduction of $C$ values. Our study reveals that single layer crystals of $\mathrm{GaS}$ and GaSe, together with their (i) high flexibility, (ii) high ductility, (iii) strain-dependent electronic behavior, and (iv) charge tunable mechanical properties, are very promising materials for nanoscale electromechanical applications.

\section{ACKNOWLEDGMENTS}

Computational resources were provided by TUBITAK ULAKBIM, High Performance and Grid Computing Center (TR-Grid e-Infrastructure). H.S. acknowledges support from Bilim Akademisi-The Science Academy, Turkey under the BAGEP program. R.T.S. acknowledges the support from TUBITAK through project $114 \mathrm{~F} 397$.
[1] B. C. Brodie, Philos. Trans. R. Soc. London 149, 249 (1859).

[2] I. K. Schuller, Phys. Rev. Lett. 44, 1597 (1980).

[3] G. R. Bhimanapati, Z. Lin, V. Meunier, Y. Jung, J. Cha, S. Das, D. Xiao, Y. Son, M. S. Strano, V. R. Cooper, L. Liang, S. G. Louie, E. Ringe, W. Zhou, S. S. Kim, R. R. Naik, B. G. Sumpter, H. Terrones, F. Xia, Y. Wang, J. Zhu, D. Akinwande, N. Alem, J. A. Schuller, R. E. Schaak, M. Terrones, and J. A. Robinson, ACS Nano 9, 11509 (2013).

[4] S. Z. Butler, S. M. Hollen, L. Cao, Y. Cui, J. A. Gupta, H. R. Gutiérrez, T. F. Heinz, S. S. Hong, J. Huang, A. F. Ismach, E. Johnston-Halperin, M. Kuno, V. V. Plashnitsa, R. D. Robinson, R. S. Ruoff, S. Salahuddin, J. Shan, L. Shi, M. G. Spencer, M. Terrones, W. Windl, and J. E. Goldberger, ACS Nano 7, 2898 (2013).

[5] S. Das, J. A. Robinson, M. Dubey, H. Terrones, and M. Terrones, Annu. Rev. Mater. Res. 45, 1 (2015).

[6] P. Miro, M. Audiffred, and T. Heine, Chem. Soc. Rev. 43, 6537 (2014).

[7] H. Sahin, E. Torun, C. Bacaksiz, S. Horzum, J. Kang, R. T. Senger, and F. M. Peeters, WIREs Inter. Rev. 6, 351 (2016).

[8] D. J. Late, B. Liu, J. Luo, A. Yan, H. S. S. R. Matte, M. Grayson, C. N. R. Rao, and V. P. Dravid, Adv. Mater. 24, 3549 (2012).

[9] X. Li, M. W. Lin, A. A. Puretzky, J. C. Idrobo, C. Ma, M. Chi, M. Yoon, C. M. Rouleau, I. I. Kravchenko, D. B. Geohegan, and K. Xiao, Sci. Rep. 4, 5497 (2014).

[10] H. Cai, J. Kang, H. Sahin, B. Chen, A. Suslu, K. Wu, F. M. Peeters, X. Meng, and S. Tongay, Nanotech. 27, 065203 (2016).

[11] P. Hu, J. Zhang, M. Yoon, X. F. Qiao, X. Zhang, W. Feng, P. Tan, W. Zheng, J. Liu, X. Wang, J. C. Idrobo, D. B. Geohegan, and K. Xiao, Nano Res. 7, 694 (2014).

[12] C. Tatsuyama, Y. Watanabe, C. Hamaguchi, and J. Nakai, J. Phys. Soc. Jpn. 29, 150 (1970).

[13] A. Yamamoto, A. Syouji, T. Goto, E. Kulatov, K. Ohno, Y. Kawazoe, K. Uchida, and N. Miura, Phys. Rev. B 64, 035210 (2001).

[14] M. Xu, T. Liang, M. Shi, and H. Chen, Chem. Rev. 113, 3766 (2013).

[15] D. J. Late, B. Liu, H. S. S. R. Matte, C. N. R. Rao, and V. P. David, Adv. Funct. Mater. 22, 1894 (2012).
[16] C. H. Ho, M. H. Hsieh, and C. C. Wu, Rev. Sci. Instrum. 77, 113102 (2006).

[17] T. Aono, K. Kase, and A. Kinoshita, J. Appl. Phys. 74, 2818 (1993).

[18] P. Hu, Z. Wen, L. Wang, P. Tan, and K. Xiao, ACS Nano 6, 5988 (2012).

[19] H. Chen, Y. Li, L. Huang, and J. Li, J. Phys. Chem. C 119, 29148 (2015).

[20] H. Chen, Y. Li, L. Huang, and J. Li, RSC Adv. 5, 50883 (2015).

[21] X. Li, L. Basile, M. Yoon, C. Ma, A. A. Puretzky, J. Lee, J. C. Idrobo, M. Chi, C. M. Rouleau, D. B. Geohegan, and K. Xiao, Angew. Chem. Int. Ed. 54, 2712 (2015).

[22] X. Zhou, J. Cheng, Y. Zhou, T. Cao, H. Hong, Z. Liao, S. Wu, H. Peng, K. Liu, and D. Yu, J. Am. Chem. Soc. 137, 7994 (2015).

[23] H. Shi, H. Pan, Y. W. Zhang, and B. I. Yakobson, Phys. Rev. B 87, 155304 (2013)

[24] J. P. Perdew, K. Burke, and M. Ernzerhof, Phys. Rev. Lett. 77, 3865 (1996).

[25] J. P. Perdew, K. Burke, and M. Ernzerhof, Phys. Rev. Lett. 78, 1396 (1997).

[26] G. Kresse and J. Hafner, Phys. Rev. B 47, 558 (1993).

[27] G. Kresse and J. Hafner, Phys. Rev. B 49, 14251 (1994).

[28] S. J. Grimme, Comput. Chem. 27, 1787 (2006).

[29] G. Henkelman, A. Arnaldsson, and H. Jonsson, Comput. Mater. Sci. 36, 354 (2006).

[30] C. Bacaksiz, H. Sahin, H. D. Ozaydin, S. Horzum, R. T. Senger, and F. M. Peeters, Phys. Rev. B 91, 085430 (2015).

[31] G.-H. Lee, R. C. Cooper, S. J. An, S. Lee, A. van der Zande, N. Petrone, A. G. Hammerberg, C. Lee, B. Crawford, W. Oliver, J. W. Kysar, and J. Hone, Science 340, 1073 (2013).

[32] C. Ataca, H. Sahin, and S. Ciraci, J. Chem. Phys. C 116, 8983 (2012).

[33] J. Kang, H. Sahin, and F. M. Peeters, Phys. Chem. Chem. Phys. 17, 27742 (2015).

[34] S. Bertolazzi, J. Brivio, and A. Kis, ACS Nano 5, 9703 (2011).

[35] C. Lee, X. D. Wei, J. W. Kysar, and J. Hone, Science 321, 385 (2008).

[36] H. Sahin, S. Cahangirov, M. Topsakal, E. Bekaroglu, E. Akturk, R. T. Senger, and S. Ciraci, Phys. Rev. B 80, 155453 (2009). 